\title{
Self-Trapping of Excitons, Violation of Condon Approximation, and Efficient Fluorescence in Conjugated Cycloparaphenylenes
}

\author{
Lyudmyla Adamska, ${ }^{\dagger}$ Iffat Nayyar, ${ }^{\dagger}$ Hang Chen, ${ }^{\ddagger}$ Anna K. Swan, ${ }^{\ddagger}$ Nicolas Oldani, ${ }^{\S}$
}

Sebastian Fernandez-Alberti, ${ }^{\S}$ Matthew R. Golder," Ramesh Jasti, "Stephen K. Doorn, ${ }^{\dagger}$ and Sergei Tretiak ${ }^{*}, \dagger$

${ }^{\dagger}$ Theoretical Division, Center for Nonlinear Studies and Center for Integrated Nanotechnologies (CINT), Los Alamos National
Laboratory, Los Alamos, New Mexico 87545, United States
${ }^{\ddagger}$ Department of Electrical and Computer Engineering, and Photonics Center, Boston University, Boston, Massachusetts 02215,
United States
${ }^{\S}$ Universidad Nacional de Quilmes, Roque Saenz Pea 352, B1876BXD Bernal, Argentina
${ }^{\|}$Department of Chemistry and Biochemistry and Materials Science Institute 1253, University of Oregon, Eugene, Oregon 97403,
United States

Supporting Information

ABSTRACT: Cycloparaphenylenes, the simplest structural unit of armchair carbon nanotubes, have unique optoelectronic properties counterintuitive in the class of conjugated organic materials. Our time-dependent density functional theory study and excited state dynamics simulations of cycloparaphenylene chromophores provide a simple and conceptually appealing physical picture explaining experimentally observed trends in optical properties in this family of molecules. Fully delocalized

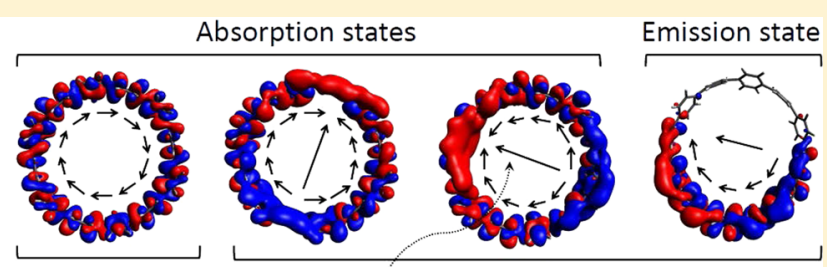

Optically forbidden

Optically active

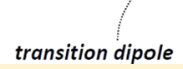
degenerate second and third excitonic states define linear absorption spectra. Self-trapping of the lowest excitonic state due to electron-phonon coupling leads to the formation of spatially localized excitation in large cycloparaphenylenes within $100 \mathrm{fs}$. This invalidates the commonly used Condon approximation and breaks optical selection rules, making these materials superior fluorophores. This process does not occur in the small molecules, which remain inefficient emitters. A complex interplay of symmetry, $\pi$-conjugation, conformational distortion and bending strain controls all photophysics of cycloparaphenylenes.

KEYWORDS: Time-dependent density functional theory, nonadiabatic excited state molecular dynamics, exciton-vibrational coupling, photoluminescence spectra

\begin{abstract}
T $\mathrm{n}$ recent years we have witnessed rapid development of nanotechnologies spanning multiple classes of materials such as organic and metal-organic structures, semiconductor nanocrystals, and metal clusters. These systems have unique properties not easily found in other materials due to competition of various degrees of freedom (e.g., electronic, vibrational, spin, and so forth), which are frequently controlled by quantum confinement mechanisms. Understanding and interpretation of these new functionalities are not readily attainable using approaches developed for molecules or for extended solids. One example of such systems is cycloparaphelynenes $([n] \mathrm{CPP}),{ }^{1}$ which consist of $n$ phenyl units connected in a conjugated periodic chain (see inset in Figure 1). Owing to their geometry, CPPs have unique physicochemical and electronic properties valuable for multifarious applications in materials science and technology such as photovoltaic, photoelectronic and light-emission technologies.

The first successful attempt of synthesis of cycloparaphenylene dates back to 1993 targeting [6]-[10]CPPs. ${ }^{2}$ In 2008, several CPPs were synthesized and characterized by Jasti et al. ${ }^{3}$
\end{abstract}

Since then, cycloparaphelynenes have been attracting a great amount of interest in the fields of synthetic chemistry, ${ }^{3-15}$ computational materials science, ${ }^{3,5,16-19}$ and fundamental physics. ${ }^{1,11,14}[n]$ CPPs of sizes 5 through 16 and 18 were synthesized with certain ring sizes produced on a gram scale. Success with cycloparaphenylenes motivated scientists to grow cyclic molecules with other building blocks, ${ }^{20-22}$ combine different building blocks in one molecule, ${ }^{10,13}$ synthesize covalently linked dimers, ${ }^{23,24}$ and tune the atomic structure of these molecules with substitutional impurities. ${ }^{20}$ One exciting application of cyclic molecules is using them as seeds for growing carbon nanotubes. ${ }^{25}$ Cycloparaphenylene is the shortest segment of an armchair nanotube. The other seeds explored by chemists are cyclacene (the shortest segment of zigzag nanotubes ${ }^{22,25}$ ) and modified CPP (a seed for chiral nanotubes $\left.{ }^{21,26}\right)$. Thus, this constitutes a promising avenue for

Received: August 14, 2014

Revised: October 3, 2014

Published: October 13, 2014 

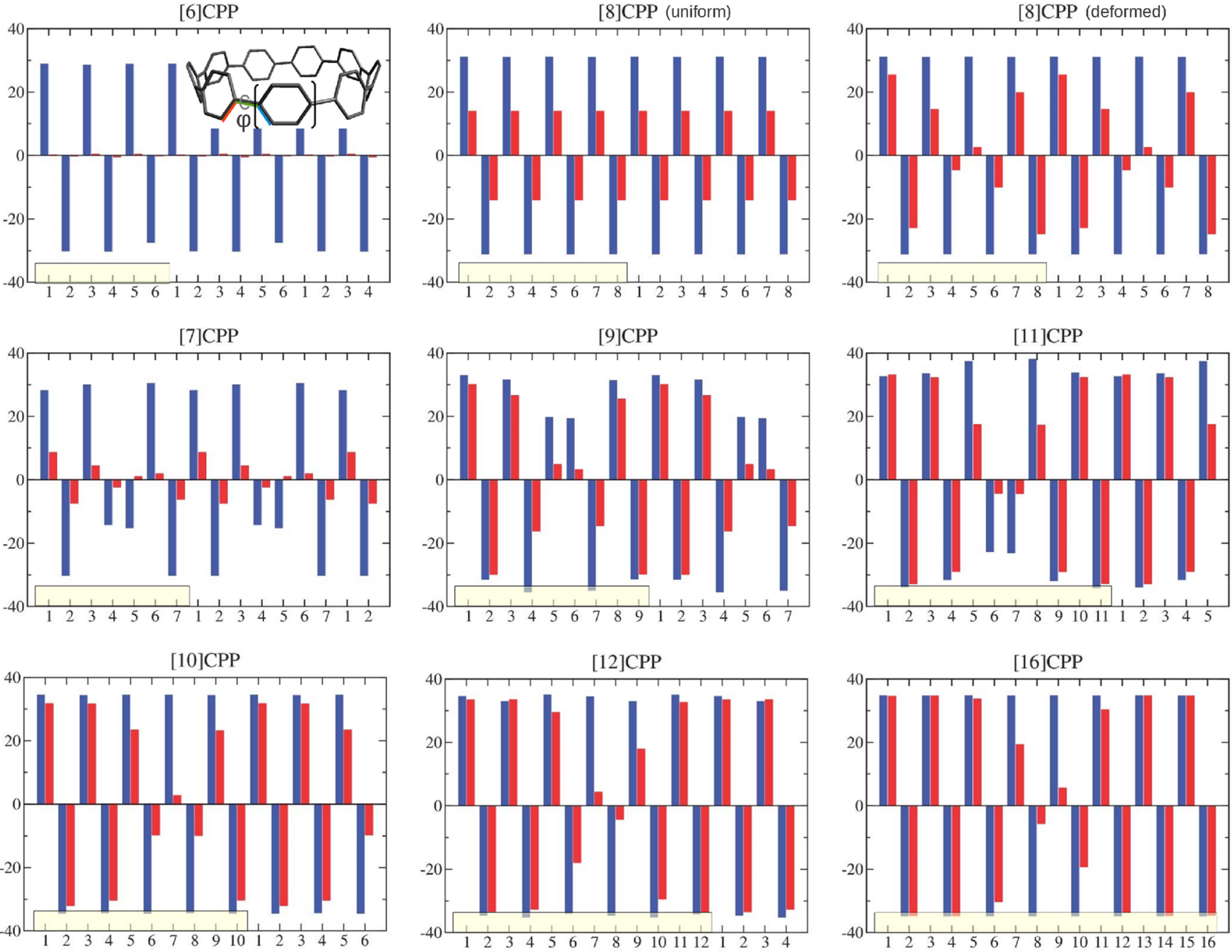

Figure 1. Variation of dihedral angles (degrees) along the chain of $[n]$ CPP molecules in the ground state (blue) and excited state (red). Yellow bar marks the length of the molecule. The inset shows chemical structure of the $[n]$ CPP hoop. Notably, [8]CPP has two excited state geometries with near-degenerate total energy minima (denoted as uniform and deformed structures).

Table 1. Calculated Wavelengths $\lambda$ and Oscillator Strength $f$ for $S_{1}, S_{2}, S_{3}$, and $S_{1^{\prime}}$ Transitions Compared to Experimentally Measured Absorption and Emission Spectral Maxima and Fluorescence Quantum Yield $\Phi_{\mathrm{F}}$ for $[n]$ CPP molecules ${ }^{a}$

\begin{tabular}{|c|c|c|c|c|c|c|c|c|c|c|c|}
\hline \multirow[b]{2}{*}{$n$} & \multicolumn{2}{|c|}{$S_{1}$} & \multicolumn{2}{|c|}{$\mathrm{S}_{2}, \mathrm{~S}_{3}$} & \multirow{2}{*}{$\frac{\text { absorption (exp.) }}{\lambda_{\mathrm{abs},}, \mathrm{nm}}$} & \multicolumn{2}{|c|}{$S_{1^{\prime}}$} & \multicolumn{2}{|c|}{ Emission (exp.) } & \multicolumn{2}{|c|}{$\tau, \mathrm{ns}$} \\
\hline & $\overline{\lambda_{\mathrm{abs}}, \mathrm{nm}}$ & $f$ & $\lambda_{\mathrm{abs}}, \mathrm{nm}$ & $f$ & & $\lambda_{\mathrm{em}}, \mathrm{nm}$ & $f$ & $\lambda_{\mathrm{em}}, \mathrm{nm}$ & $\Phi_{\mathrm{F}}$ & this work & $\exp ^{c}$ \\
\hline 6 & 381 & 0.00 & 297 & 1.16 & & 679 & 0.00 & & $0.0^{e}$ & & \\
\hline 7 & 374 & 0.03 & 300 & 1.56 & $340^{h}$ & 563 & 0.002 & $588^{h}$ & $0.007^{e}, 0.006^{h}$ & 1587 & \\
\hline 8 & 346 & 0.00 & 300 & 1.88 & $338^{c}, 340^{d}$ & 484 & 0.44 & $533^{c}, 531^{d}$ & $0.084^{c}, 0.1^{e}$ & 5.63 & 17.6 \\
\hline 9 & 344 & 0.07 & 301 & 2.22 & $339^{b}, 340^{c, f, i}, 341^{d}$ & 464 & 0.89 & $494^{b, c, i}, 499^{d}, 500^{f}$ & $0.73^{b}, 0.30^{c}, 0.38^{e}$ & 2.53 & 10.6 \\
\hline 10 & 331 & 0.00 & 301 & 2.57 & $340^{c}, 341^{d}$ & 452 & 1.20 & $470^{c}, 474^{d}$ & $0.46^{c}, 0.65^{e}$ & 1.79 & 6.6 \\
\hline 11 & 331 & 0.10 & 302 & 2.90 & $339^{c}, 340^{d}$ & 445 & 1.45 & $458^{c}, 460^{d}$ & $0.52^{c}, 0.73^{e}$ & 1.44 & 3.8 \\
\hline 12 & 324 & 0.00 & 303 & 3.26 & $338^{b, c}, 339^{d, f}, 340^{i}$ & 440 & 1.67 & $450^{b, c, d, i}, 452^{f}, 464^{g}$ & $0.89^{b}, 0.66^{c}, 0.81^{e}$ & 1.22 & 2.7 \\
\hline 16 & 318 & 0.00 & 304 & 4.64 & $339^{b_{f} f}, 338^{d}$ & 423 & 2.31 & $438^{b_{f} f}$ & $0.88^{b}$ & 0.83 & \\
\hline
\end{tabular}

${ }^{a}$ Calculated radiative lifetimes $\tau$ are also shown in comparison with experimental data. States $S_{2}$ and $S_{3}$ are degenerate for even $n$ and approximately degenerate for odd $n(\Delta \lambda<5 \mathrm{~nm}, \Delta f<0.01)$; this table lists numbers corresponding to $S_{3} .{ }^{b}$ Reference $5 .{ }^{c}$ Reference 13 . ${ }^{d}$ Reference $15 .{ }^{e}$ Reference 6 . $f_{\text {Reference } 12 .{ }^{g} \text { Reference } 21 .{ }^{h} \text { Reference } 10 .{ }^{i} \text { Reference } 3 .}$

synthesizing well-defined nanotube segments spanning a transition between the molecular regime and the more condensed matter behavior of the extended nanotube structures.

The structural and photophysical properties of $[n]$ CPPs have been intensely studied both experimentally $5,6,12,13,15$ and theoretically. ${ }^{5,17-19}$ Cycloparaphenylenes have been optically characterized both in solution and in the solid state (van der Waals crystal and films). These molecules have high optical absorbance in a blue spectral region that increases with the ring size, however, the absorption maxima (at $\sim 340 \mathrm{~nm}$ ) are independent of the ring size. In contrast, the fluorescence 
[8]-CPP

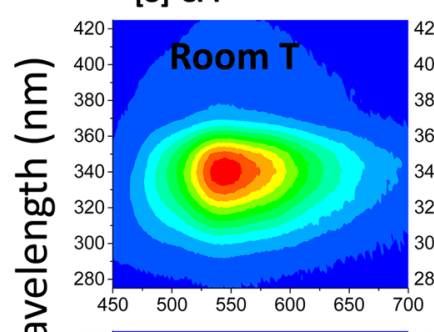

[9]-CPP
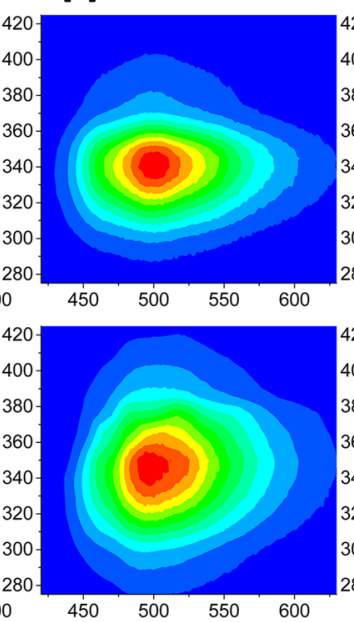

[10]-CPP

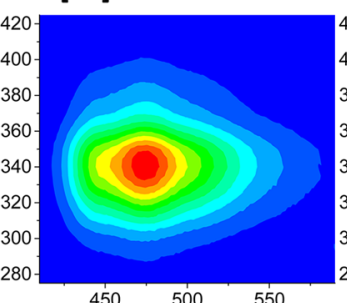

450

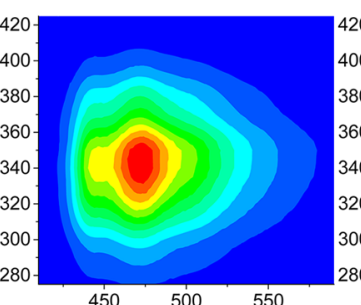

[11]-CPP

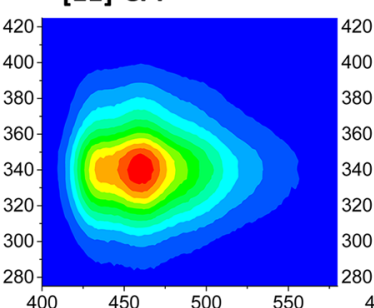

[12]-CPP

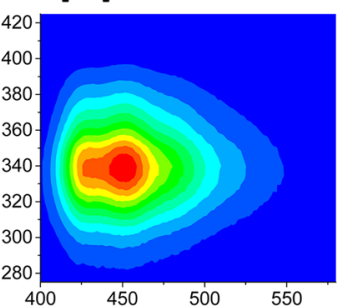

\section{Emission Wavelength $(\mathrm{nm})$}

Figure 2. PLE spectra of [8]-[12]CPP molecules at room temperature (top row) and at $77 \mathrm{~K}$ (low T, bottom row). Photoluminescence intensity is shown as a function of excitation and emission wavelengths. Low-temperature spectra allow resolution of vibronic features for larger rings.

maxima become strongly red shifted with decrease of molecular size. Moreover, larger hoops are shown to be excellent emitters, whereas small CPPs have very poor/vanishing fluorescence quantum yield. Table 1 summarizes the reported experimental photophysical properties of $[n]$ CPPs. Furthermore, Figure 2 provides detailed experimental information on absorption and emission in several hoops at room and low $(77 \mathrm{~K})$ temperatures in the form of two-dimensional plots (see slices of these plots on the same wavelength scale in Supporting Information Figure $2 \mathrm{~S}$,). Interestingly, these cyclic molecules may also host few electrons, for example, [8]CPP is able to accept four electrons without breaking the hoop. ${ }^{14}$ Such broad size-tunability of the optoelectronic properties of $[n]$ CPPs make them attractive for many applications based on organic semiconductor carbon materials.

Despite the large body of previous work, however, a detailed understanding of the relationships between the underlying structures (e.g., backbone strain, steric interactions, symmetry, and aromatic conjugation) and molecular optical properties is still missing. Reported results for most previous computational efforts (e.g., refs 5 and 17-19) have shown strong dependence on the methodology (e.g., DFT model) used. While probably most accurate, GW simulations of excited state properties of $\mathrm{CPPs}^{3}$ did not provide analysis of electronic wave functions at absorption and emission points. Subsequently, experimental size-dependent trends in excited state energies (particularly emission wavelengths) and optical selection rules were poorly reproduced and explained. Herein, we present a timedependent density functional theory (TD-DFT) study and excited state molecular dynamics simulations that capture essential features of the experimental absorption and emission spectra and suggest physically simple rationale for the observed trends in connection to molecular geometry. Our results suggest that the photophysics of $[n]$ CPPs is dictated by a complex dynamics of excitons (a bound state of electron-hole pair) on a circle strongly coupled to vibrational degrees of freedom, which may lead to exciton localization (self-trapping) on a length scale of about five rings in large molecules but is not attainable in small systems.
Ground State Geometry. We start our discussion with the geometry of $[n]$ CPP molecule being a result of a complex interplay between steric interactions (which tend to increase dihedral angles between neighboring phenyls), $\pi$-conjugation (which tends to planarize the chain) and backbone strain (which frustrates the above interactions and mixes $\sigma$ - and $\pi$ bonds between neighboring rings). In agreement with previous studies of the ground state geometry of $[n] \mathrm{CPP}$ molecules, ${ }^{3,16,17}$ our results show the optimal dihedral angle between neighboring phenyls to be about $30^{\circ}$. Thereby, molecules with an even number of phenyl units are able to achieve this optimal configuration by alternating the dihedral along the chain as shown in Figure 1 (blue bars) and Figure 3a ([6]CPP and $[12] \mathrm{CPP})$. An increase of the backbone $\operatorname{strain}^{5}$ that is pronounced in smaller hoops tends to decrease dihedrals, thus increasing $\pi$-conjugation. For example, $[16] \mathrm{CPP}$ and, [6] CPP have dihedral angles of $\pm 36^{\circ}$ and $\pm 28^{\circ}$, respectively. In contrast, the odd-numbered $[n]$ CPPs cannot adopt such high symmetry configuration. These molecules are examples of frustrated structures containing a defect, that is, one of the rings is connected to its neighbors by dihedral angles of about 15$20^{\circ}$. Notably, our calculations show that a defect localized on three rings is energetically preferable compared to the other possible geometries. This is visualized in Figure 1 (blue bars) and Figure 3a ([9]CPP).

Absorption Spectra. To discuss the CPPs' linear absorption properties, we first recall that electronic excitations in conjugated oligomers and polymers have an excitonic nature with strong binding energy between photoexcited electron and hole. $^{27-29}$ The lowest excitonic (band gap) state $S_{1}$ (see Methods) typically "collects" nearly all oscillator strength from its parent band, making these materials excellent light absorbers and emitters. To rationalize the above in linear chains, we invoke simple physical considerations from the exciton scattering model. ${ }^{29}$ Namely, let an exciton be a "quasiparticle" placed in a box (limited by molecular ends or chain defects). The resulting excitonic envelope wave functions forming a band are then standing waves with an increasing number of nodes and an increase of energy, which correspond to states with different momenta in the infinite chain limit. ${ }^{29}$ The transition 
(a) Atomic Structure

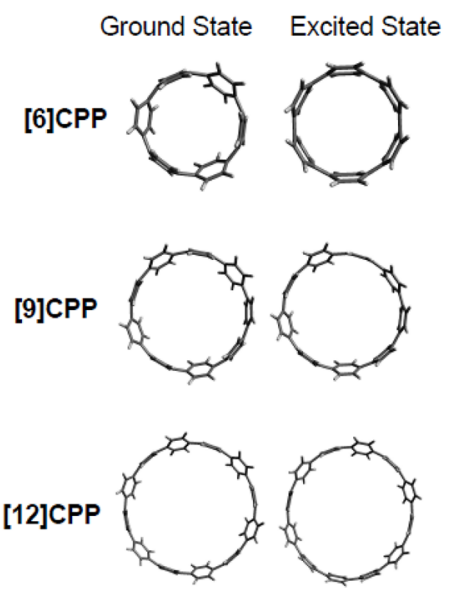

(c)
Transition Dipole (sketch for [12]CPP)

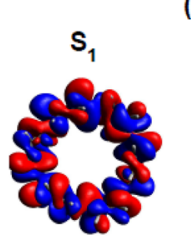

(b) Transition Density
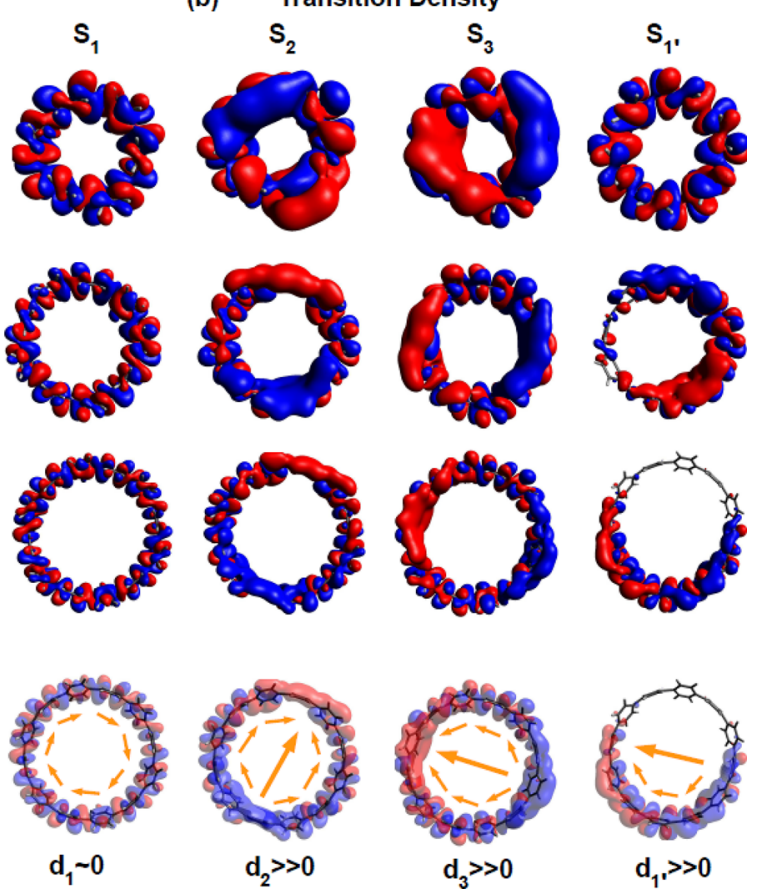

Figure 3. (a) Structure of [6]-, [9]-, and [12]CPP molecules demonstrating conformational differences in the ground and excited states. (b) Orbital distribution of transition density for $S_{1-3}$ and $S_{1^{\prime}}$ transitions in [6]-, [9]-, and [12]CPPs. The corresponding frequencies and oscillator strengths are listed in Table 1. (c) Schematic of transition dipole shown for $S_{1-3}$ and $S_{1^{\prime}}$ transitions in [12]CPP molecule. Red and blue color corresponds to negative and positive values of the transition density, respectively.

dipole moment (and respective oscillator strength) of each discrete state can be roughly represented as a vector superposition of effective dipoles of each repeat unit, where the sign of the wave function lobe defines the elementary dipole directions. ${ }^{30}$ Subsequently, in a linear chain the nodeless $S_{1}$ state attains the largest dipole (all constructive superposition), the one-mode state $S_{2}$ is optically forbidden (the destructive superposition between the right and left lobes), and so on. ${ }^{29}$

The same framework can be applied to the CPPs but it needs to account for closed boundary conditions and circular geometry. Similar to the linear chain, the lowest $S_{1}$ state is node-less and delocalized across the entire molecule as shown by examining the transition density plots for several hoops in Figure $3 b$. The transition density characterizes changes in the ground state electronic density across the molecule upon excitation from the ground to a specific excited state, and, therefore, directly defines the value of the transition dipole moment. Subsequently, in contrast to the linear chain $S_{1}$ is optically forbidden in circular geometries as pictorially shown in Figure 3c. Indeed, our direct calculations result in a vanishing (very weak) $S_{1}$ oscillator strength in even- (odd-) numbered [n]CPPs (see Table 1). Of course, imperfect geometry of CPPs with odd rings leads to small perturbations in the electronic wave function which results in small but nonzero oscillator strength. While $S_{1}$ is forbidden in the linear response, it can be probed by nonlinear spectroscopies such as two-photon absorption, where the selection rule restrictions are lifted. This was recently experimentally achieved by Itami and coworkers. ${ }^{12}$

Energies of the next electronic states $S_{2}$ and $S_{3}$ are degenerate (quasi-degenerate) in even- (odd-) numbered [ $n]$ CPPs due to symmetry reasons. Their wave functions (Figure $3 \mathrm{~b}$ ) have 2 nodes on opposite sides of the ring. Circular geometry ensures constructive superposition of elementary dipoles as illustrated in Figure 3c. Subsequently, $S_{2}$ and $S_{3}$ states are strongly optically allowed with perpendicular polarization emerging from the orthogonal transition dipole moments, making the CPPs good absorbers. As expected for conjugated molecules, their net oscillator strength (and optical absorption) quickly grows with increasing $\mathrm{n}$ in agreement with numerous experiments (Table 1). Our calculations also find that transition energies of $S_{2}$ and $S_{3}$ states do not depend on the molecular size, thus reproducing experimental data (Table 1 ). This is a consequence of several factors: (i) transition energies exhibit red shifts with an increase of ring size commensurating with a behavior of $S_{1}$ state in linear conjugated oligomers of increasing lengths; ${ }^{18,29}$ (ii) the splitting between $S_{1}$ and $S_{2} / S_{3}$ states decreases with an increase of $n$ (i.e., both (i) and (ii) being "larger box size" effects); and (iii) smaller CPPs have better $\pi$ conjugation on/between neighboring phenyl rings due to reduced dihedrals, backbone strain, and through-space interactions (the latter effects on the frontier molecular orbital energies have been investigated in detail in ref 16). Subsequently, nearly exact cancelation of (i)/(ii) (red shifts with an increase of $n$ ) and (iii) (blue shifts with an increase of $n$ ) makes absorption maxima (i.e., positions of $S_{1}$ and $S_{2} / S_{3}$ states) to be virtually independent of the CPP hoop size. Energy of $S_{1}$ transition weakly red shifts for smaller hoops, where effect (iii) is getting stronger. As it is a frequent case of use of range-corrected DFT models, ${ }^{27,28}$ calculated values of all state transition energies are uniformly blue-shifted by about 40 $\mathrm{nm}$ compared to the respective experimental numbers (Table 1). The natural defect in odd-numbered CPPs results in a small asymmetry between $S_{2}$ and $S_{3}$ states: difference in wavelength, corresponding to these excited states, is less than $5 \mathrm{~nm}$. Notably, the experimental absorption profiles of all [8][12] CPP rings (Figure 2) remain centered at about $340 \mathrm{~nm}$ at both room and low temperatures. Particularly, in contrast to 
many other conjugated systems, it is remarkable that none of these excitation spectra at low temperature exhibit vibrational features: all spectra are broad and single-peaked with a width of about $60 \mathrm{~nm}$ (small hoops) to $80 \mathrm{~nm}$ (large hoops) similar to their counterparts at room temperature. This suggests inhomogeneous broadening due to conformational structural variations (aggregation effects were excluded for our experimental conditions). Indeed, a previous DFT study ${ }^{16}$ suggested the existence of multiple ground state conformers in CPP systems due to irregular variations of the dihedral angles (regular alternating geometry being the lowest energy structure). These conformers differ in the ground state energy by $1-3 \mathrm{kcal} / \mathrm{mol}$, have transition barriers of less than $4 \mathrm{kcal} /$ mol, ${ }^{16}$ and affect transition energies to $S_{2}$ and $S_{3}$ states by up to $30-40 \mathrm{~nm}$ as verified by our selective TD-DFT simulations. Subsequently, it is likely that static torsional disorder provides a major contribution to the width of absorption spectra across the broad temperature range. Frequently, circular molecular aggregates have similar optical selection rules for excitonic manifold. Absorption and fluorescence properties of such analogous systems in the presence of weak perturbations have been previously extensively explored using Frenkel-exciton tight-binding models. ${ }^{31,32}$

Excited State Geometry. Photoexcitation of an electronhole pair results in a decreased dimerization (i.e., difference between effective single $\mathrm{C}-\mathrm{C}$ and double $\mathrm{C}=\mathrm{C}$ bonds) of $\pi$ electronic density in conjugated systems. This frequently leads to a self-trapped localized excitonic state characterized by reduced bond-length alternation and more planar conformations, which appears in emission. ${ }^{33}$ These effects have been previously explored both experimentally and theoretically in the case of poly(phenylene-vinylene)s and polyfluorenes. ${ }^{33-36} \mathrm{~A}$ similar phenomenon is observed in the CPP case as well. As one may see in Figure 1 (red bars), CPPs with $n>8$ indeed develop a local planarization in the $S_{1^{\prime}}$ state (prime stands for excited state calculated in the optimal excited state geometry, see Methods) on the length of about 5 rings, while preserving the ground state geometry on the rest of the rings, which indirectly indicates a placement and self-trapping of the excitonic wave function on the distorted portion of the hoop. In contrast, the $S_{1^{\prime}}$ geometry in[6]CPP and[7]CPP hoops has uniformly vanishing dihedrals due to small molecular size and effects of strong backbone strain favoring planar configuration. [8] CPP is an intermediate case showing both geometry minima (see Figure 1, top row) having only $0.027 \mathrm{eV}$ absolute energy difference. [9] CPP and larger hoops show localization in the excited state. We further note that B3LYP functional and lack of solvent model mostly used in the previous TD-DFT calculations of CPPs ${ }^{5,13,17}$ proved to be deficient to model experimentally observed excitation localization phenomena in conjugated polymers and was unable to reproduce deformed structures $^{27,28}$ appearing due to exciton self-trapping. ${ }^{33-36}$ Subsequently, previously reported results for excited state geometries are qualitatively different from ours (e.g., see Supporting Information Table S1 and Figure S1).

Fluorescence Properties. According to the celebrated Kasha's rule, ${ }^{37}$ molecular fluorescence generally occurs from the lowest singlet excited state $S_{1^{\prime}}$. By examining experimental emission profiles in [8]-[12] CPP rings (Figure 2), we notice a lack of vibrational progression in all plots taken at the room temperature. At low temperature, vibrational features (separated $0-0$ and $0-1$ lines) are clearly appearing only for the largest $[11] \mathrm{CPP}$ and [12] CPP hoops and are completely absent in the smallest [8] CPP and [9] CPP rings. The separations in vibronic progression of about $1300 \mathrm{~cm}^{-1}$ seem to correlate with $\mathrm{C}-\mathrm{C}$ stretching vibrational modes probed by Raman spectroscopy in detail. ${ }^{11}$ Our calculations estimate the Huang-Rhys factor for this vibrational mode to be about one, whereas the integrated Huang-Rhys factor across the entire vibrational manifold is about 2-3 depending on the molecule. Such vibrational couplings are typical for conjugated chromophores. $^{33-35}$ Bending in smaller hoops seems to enhance effective vibrational coupling (thus reducing intensity of the $0-$ 0 transition) and couple more vibrational modes including out of plane phenyl ring stretching and bending, which contribute to the line broadening. Apart from vibronic features, we do not observe significant shifts for emission maxima when comparing room- and low-temperature data for any given CPP molecule. This contradicts previously predicted large temperature effects for emission in the computational study ${ }^{19}$ attributing efficient fluorescence of CPP to Jahn-Teller distortion effects. Our computational results reproduce very well experimental red shifts of emission wavelength with decreasing molecular size. This is attributed to increased $\pi$-conjugation (more planar and bent structures) and developing "though-space" orbital interactions observed in small CPP hoops. Thus, both experimental and theoretical modeling suggest significant Franck-Condon activity in all CPPs due to slow (torsional) and fast $(\mathrm{C}-\mathrm{C}$ stretching and bending) modes as is the case for many other conjugated systems. ${ }^{27,33-35}$

Most notably, our calculated excited state geometry distortion signifies exciton self-trapping in large CPPs due to vibronic coupling (see $S_{1^{\prime}}$ plots for [9] CPP and [12]CPP in Figure $3 \mathrm{~b}$ ). Such localization leads to an appearance of a significant transition dipole moment (and oscillator strength, see Table 1) during excited state equilibration $\left(S_{1} \rightarrow S_{1^{\prime}}\right)$. This strongly violates the commonly used Condon approximation ${ }^{38}$ stating that the dipole should be independent of the vibrational coordinates. Such excited state vibronic processes thus make large CPPs to be excellent fluorescent chromophores with quantum yields well exceeding $50 \%$ as observed in many experiments (Table 1). The small [6]CPP and [7]CPP hoops, which do not develop such self-trapping, have vanishing transition dipole moment for the $S_{1^{\prime}}$ state (see [6]CPP plot in Figure $3 b$ ). This agrees well with very weak fluorescence efficiency in experimental samples (Table 1). [8]CPP is a borderline due to its quasi-degenerate excited state minima (Table 1). Indeed, the experimental fluorescence quantum yield of [8]CPP is about $10 \%$, being significantly smaller than that of larger hoops. Experimentally measured radiative lifetimes ${ }^{13}$ (summarized in Table 1) can be directly compared to calculations. Transition energies $E$ and respective oscillator strengths $f$ allow evaluating radiative lifetimes $\tau$ as $\tau=\left(\hbar^{4} c^{3}\right)$ $\left.2 n E^{2} f\right)$, where $n$ is the refractive index of the solvent (1.42 for dichloromethane), $\hbar$ is the Planck's constant, and $c$ is the speed of light. As established by previous studies, ${ }^{39}$ calculations underestimate experimental lifetimes by a factor $2-3$. This is attributed to overestimated calculated values of both transition energies and transition dipole moments as well as vibrational progression distributing dipolar strength over multiple electronvibrational transitions (which was not accounted for in our simple theoretical estimates). Nevertheless, calculated radiative lifetimes reproduce the experimental trends very well.

Excited State Dynamics. Our conclusions attributing efficient fluorescence in large CPP hoops to a broken Condon approximation due to exciton self-trapping is based on the 
"static" DFT/TD-DFT calculations addressing just the minima of ground and excited state potential energy surfaces. This scenario is different from a variety of other hypotheses proposed in the literature explaining emission from the forbidden $S_{1}$ state to phonon-assisted transitions, ${ }^{12}$ vibrational intensity borrowing from the higher states, ${ }^{18}$ and Jahn-Teller distortion effects due to coupling to circle-to-oval vibrational modes breaking the selection rules. ${ }^{19}$ To verify our DFT results, we have conducted nonadiabatic excited state dynamics (NA-ESMD) simulations of internal conversion to $S_{1}$ (emission point) after photoexcitation to $S_{2} / S_{3}$ states (absorption point) in several $[n]$ CPPs at room temperature by propagating 500 trajectories to achieve statistical sampling (see Methods). Figure 4a shows that internal conversion completes within 200

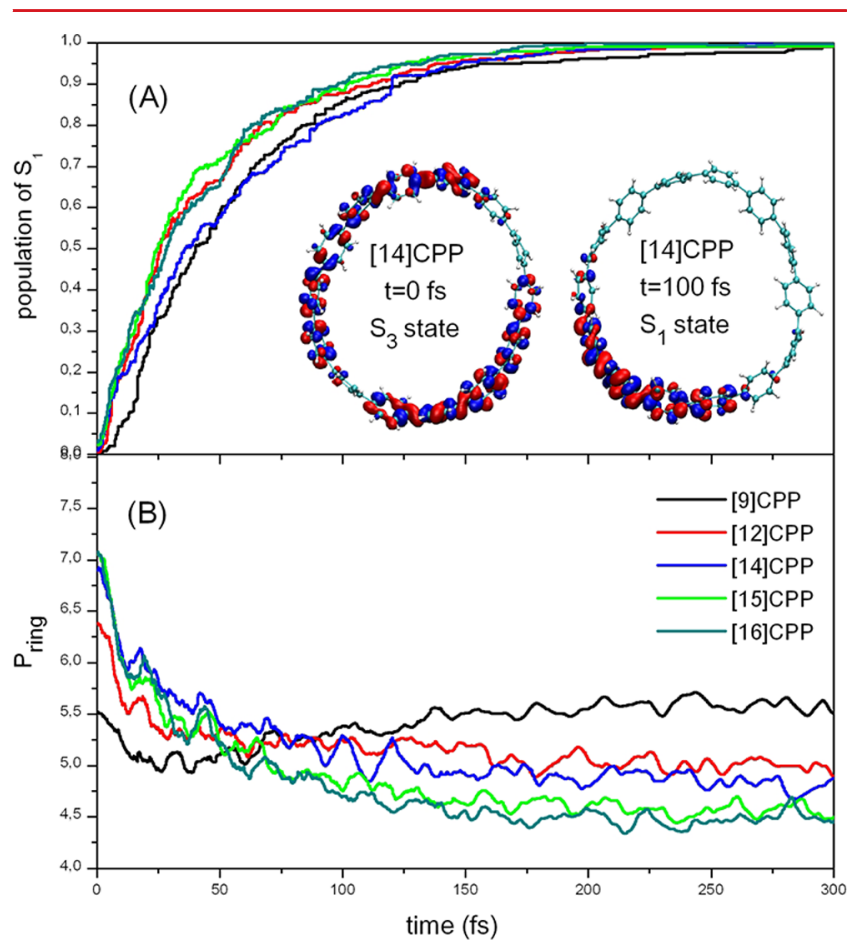

Figure 4. Nonadiabatic dynamics simulations of internal conversion at room temperature $T=300 \mathrm{~K}$ in [9]-, [12]-, [14]-, [15]-, and [16]CPP molecules after an excitation to $S_{2}$ and $S_{3}$ states. (a) Percentage of population in $S_{1}$ state calculated from the number of trajectories in it at a given time showing rapid relaxation within $200 \mathrm{fs}$ in all CPP molecules; (b) time-dependence of the average participation number of phenyl ring demonstrating ultrafast spatial localization of photoexcitation within 50 fs.

fs in all systems, being slightly faster in the larger hoops due to smaller $S_{2} / S_{1}$ gaps. Transition density plots for all trajectories (representative examples are shown as insets in Figure 4a) at the beginning and end of the dynamics have exactly the same structures as those calculated with TD-DFT for $S_{2} / S_{3}$ (delocalized exciton) and $S_{1^{\prime}}$ (self-trapped exciton) states in Figure 3, respectively. A quantitative measure of excitonic delocalization is provided by participation ratio $P_{\text {ring }}$ showing on average over how many phenyl rings the excitonic wave function is delocalized. Even in the ideal uniform geometry, the wavefuction is distributed nonuniformly over carbon atoms of the phenyl ring, so that $P_{\text {ring }}$ is always smaller than the number of phenyls in the CPP hoop (e.g., in [14]CPPs $P_{\text {ring }}^{S_{1}}=7.8, P_{\text {ring }}^{S_{2}}$ $=P_{\text {ring }}^{\mathrm{S}_{3}}=8.4$ at the ground state geometry). Room-temperature thermal fluctuations effectively reduce $P_{\text {ring }}$ even further. Figure
$4 \mathrm{~b}$ shows the calculated time-evolution of this quantity for the NA-ESMD ensemble in different $[n]$ CPPs. This plot exemplifies that within $50 \mathrm{fs}$ excitation becomes localized from about 7 to 5 rings in all large hoops, which fully agrees with our static TD-DFT simulations. In contrast, this localization is much less pronounced in smaller systems. Such ultrafast localization is attributed to nonadiabatic transitions between excited states. More detailed nonadiabatic dynamics simulations ${ }^{36}$ along with time-resolved spectroscopic probes of CPP materials studying photoinduced pathways and time scales of these dynamics, will be reported elsewhere.

Conclusions. Our quantum-chemical DFT/TD-DFT simulations, nonadiabatic excited state dynamics modeling and experimental temperature-resolved absorption/emission data bring a new level of understanding to the electronic structure and photophysical properties in conjugated cycloparaphenylenes. An efficient fluorescence in large $[n]$ CPP hoops is assigned to a spatial localization of exciton (self-trapping) due to strong vibronic coupling, which breaks the Condon approximation and overrides the optical selection rules. This process is not feasible in the small $[n]$ CPP systems where the wave function remains always delocalized because of bending and strain. Our excited state dynamics simulations fully confirm this scenario and estimate a time scale of excitonic localization to be about 50 fs. These computational results establish a firm connection of photophysical properties with the complex localization and delocalization dynamics of excitonic states controlled by conformational structure and strong electronphonon coupling present in these chromophores. This provides valuable insights into photophysics and guidelines for future synthetic efforts in circular nanostructure systems aiming to control light-induced dynamics and energy/charge transfers.

Methods. Quantum Chemistry Calculations. All electronic structure calculations were performed using Gaussian 09 suite $^{40}$ with $6-31 \mathrm{G}^{*}$ basis set and the Coulomb-attenuated hybrid B3LYP (CAM-B3LYP) functional. ${ }^{41}$ The effect of solvent was included in a flavor of the conductor-like polarizable continuum model (CPCM) using experimentally relevant dichloromethane solvent $\left(\mathrm{CH}_{2} \mathrm{Cl}_{2}, \varepsilon=9.02\right)$ as implemented in Gaussian09 package. ${ }^{40}$ Such choice of the model chemistry has been argued by previous TD-DFT studies of conventional conjugated polymers. ${ }^{27,28,42,43}$ It was found that experimentally observed localized excitonic and charged (polaronic) states with concomitant structural distortion can be modeled only with the use of range-corrected hybrid DFT models including longrange electronic exchange interactions (e.g., CAM-B3LYP, LCwPBE, etc.). In contrast, pure (e.g., LDA), semilocal GGA (e.g., $\mathrm{PBE}$ ), and hybrid DFT models with small amount of orbital exchange (e.g., B3LYP (20\%) or PBE0(25\%)) result in spatially delocalized excited states. Moreover, inclusion of polarization properties of the surrounding media (i.e., solvent model) is found to be critical as well. ${ }^{28}$ Specifically, as used in the current study, the CAM-B3LYP model is found to adequately describe excitonic and charged states as well as electron-phonon coupling in the similar families of conjugated polymers and oligomers providing result similar to other range-separated models. ${ }^{27,28,44}$

The calculations of $[n]$ CPPs $(n=6,7,8,9,10,11,12$, and 16) were done in three steps. First, the ground singlet state $\left(S_{0}\right)$ geometries of CPPs were optimized with DFT. Next, several singlet excited states were calculated for each molecule using TD-DFT framework applied to ground-state geometries. Relevant are the first three lowest excited states labeled as $S_{1}$, 
$S_{2}$, and $S_{3}$. Their transition energies and respective oscillator strength characterize the optical absorption spectra. In a third step, to obtain fluorescent properties the optimal lowest excited state geometry (hereafter denoted as $S_{1^{\prime}}$ ) of each CPP was calculated with TD-DFT. Its transition energy and oscillator strength define the vertical emission wavelength and efficiency, respectively. Calculated quantities along with respective experimental data are summarized in Table 1. All optimized geometries of ground and excited state are verified global minima (e.g., for the ground state structures are similar to those reported in ref 16). Finally, to analyze the spatial extent of photoexcited wave functions of $S_{1}, S_{2}, S_{3}$, and $S_{1^{\prime}}$ states, the respective transition densities have been visualized in real space using Avogadro software. ${ }^{45}$ The orbital projection of the transition density matrix was calculated by extracting the diagonal of the transition density matrix for particular excited state and plotting this vector in a basis of local combination of atomic orbitals. These orbital plots in Figure $3 \mathrm{~b}$ are shown in perspective view. The spatial distribution of the complete transition density matrices in CPP systems has been first analyzed in ref 18.

Nonadiabatic Excited State Molecular Dynamics (NAESMD) Simulations. The NA-ESMD approach combines molecular dynamics with quantum transitions with "on the fly" analytical calculations of excited state energies, gradients, and nonadiabatic coupling terms. The method has been specifically developed to simulate photoinduced dynamics in large organic conjugated molecules involving multiple coupled electronic excited states. ${ }^{36,46,47}$ In brief, the DFT geometries of $[n]$ CPPs $(n=9,12,14,15$, and 16) were employed as our initial guess for a molecular dynamics simulation in the electronic ground state. First, for every molecule 1 ns of ground state molecular dynamics simulations at $300 \mathrm{~K}$ has been performed using a Langevin friction coefficient of $20.0 \mathrm{ps}^{-1}$. This value was chosen to mimic the solvent $\left(\mathrm{CH}_{2} \mathrm{Cl}_{2}\right)$ used in the experiments. Snapshots of 500 nuclei positions and momenta (configurational space) have been collected from the last $0.6 \mathrm{~ns}$ and used as initial conditions for the subsequent NA-ESMD simulations. The NA-ESMD trajectories have been started from these initial configurations by instantaneously promoting the system from the ground state to $S_{2}$ or $S_{3}$ initial excited state. The NA-ESMD trajectories of $500 \mathrm{fs}$ duration are propagated at $300 \mathrm{~K}$ in order to obtain a reasonable statistics. At the end of the simulations (500 fs) all trajectories have ended up in the $S_{1}$ state corresponding to the emission point. Classical time steps of 0.5 and $0.1 \mathrm{fs}$ have been used for nuclei propagation in the ground and excited state dynamics, respectively. Besides, a quantum time step of $0.025 \mathrm{fs}$ has been used to propagate the electronic degrees of freedom during the NA-ESMD simulations. More details concerning the NA-ESMD implementation, limitations, advantages, and parameters can be found elsewhere. ${ }^{36,46,47}$ Average relaxation dynamics of an ensemble of the trajectories defines growth of populations at the $S_{1}$ state (i.e., internal conversion process), which is shown in Figure 4a. Finally, at each time step and for every trajectory we have calculated the participation number using the spatial distribution of the excited state transition density. ${ }^{47}$ This quantity averaged across the ensemble quantifies an effective number of phenyl rings across which the excited state wave function is delocalized (Figure 4b).

Experimental Methods. Photoluminescence excitation (PLE) spectra were obtained on a JY-Horriba Fluorolog system from samples dissolved in $\mathrm{CH}_{2} \mathrm{Cl}_{2}$. The average $\mathrm{CPP}$ concentration was $2.2 \mu \mathrm{M}$. Low-temperature $(77 \mathrm{~K})$ spectra were obtained from samples immersed in liquid nitrogen. Emission spectra were obtained in $2 \mathrm{~nm}$ steps, while excitation spectra used $5 \mathrm{~nm}$ steps with $0.5 \mathrm{~nm}$ bandpass in both cases.

\section{ASSOCIATED CONTENT}

\section{S Supporting Information}

Additional computational results and plots of natural transition orbitals for excited states. This material is available free of charge via the Internet at http://pubs.acs.org.

\section{AUTHOR INFORMATION}

\section{Corresponding Author}

*E-mail: serg@lanl.gov.

\section{Notes}

The authors declare no competing financial interest.

\section{ACKNOWLEDGMENTS}

L.A. acknowledges Professor A. Zhugayevych for his help with data processing. This work was supported by the U.S. Department of Energy and Los Alamos LDRD funds. This work was also partially supported by CONICET, UNQ, ANPCyT (PICT-2010-2375). Los Alamos National Laboratory is operated by Los Alamos National Security, LLC, for the National Nuclear Security Administration of the U.S. Department of Energy under contract DE-AC5206NA25396. We acknowledge support from the Center for Integrated Nanotechnologies (CINT) and the Center for Nonlinear Studies (CNLS) at LANL.

\section{REFERENCES}

(1) Evans, P. J.; Jasti, R. Molecular Belts; Topics in Current Chemistry; Springer: Berlin, 2013; pp 1-42.

(2) Friederich, R.; Nieger, M.; Vögtle, F. Chem. Ber. 1993, 126, $1723-1732$.

(3) Jasti, R.; Bhattacharjee, J.; Neaton, J. B.; Bertozzi, C. R. J. Am. Chem. Soc. 2008, 130, 17646-17647.

(4) Evans, P. E.; Darzi, E. R.; Jasti, R. Nat. Chem. 2014, 6, 404-408.

(5) Segawa, Y.; Fukazawa, A.; Matsuura, S.; Omachi, H.; Yamaguchi, S.; Irle, S.; Itami, K. Org. Biomol. Chem. 2012, 10, 5979-5984.

(6) Darzi, E. R.; Sisto, T. J.; Jasti, R. J. Org. Chem. 2012, 77, 66246628.

(7) J.Sisto, T.; Golder, M. R.; Hirst, E. S.; Jasti, R. J. Am. Chem. Soc. 2011, 133, 15800.

(8) Hirst, E. S.; Jasti, R. J. Org. Chem. 2012, 77, 10473-10478.

(9) Xia, J.; Jasti, R. Angew. Chem., Int. Ed. 2012, 51, 2474-2476.

(10) Li, P.; Sisto, T. J.; Darzi, E. R.; Jasti, R. Org. Lett. 2013, 16, 182185.

(11) Chen, H.; Golder, M. R.; Wang, F.; Jasti, R.; Swan, A. K. Carbon 2013, 67, 203-213.

(12) Nishihara, T.; Segawa, Y.; Itami, K.; Kanemitsu, Y. J. Phys. Chem. Lett. 2012, 3, 3125-3128.

(13) Fujitsuka, M.; Cho, D. W.; Iwamoto, T.; Yamago, S.; Majima, T. Phys. Chem. Chem. Phys. 2012, 14, 14585-14588.

(14) Zabula, A. V.; Filatov, A. S.; Xia, J.; Jasti, R.; Petrukhina, M. A. Angew. Chem., Int. Ed. 2013, 52, 5033-5036.

(15) Iwamoto, T.; Watanabe, Y.; Sakamoto, Y.; Suzuki, T.; Yamago, S. J. Am. Chem. Soc. 2011, 133, 8354-8361.

(16) Segawa, Y.; Omachi, H.; Itami, K. Org. Lett. 2010, 12, 22622265.

(17) Sundholm, D.; Taubert, S.; Pinchierri, F. Phys. Chem. Chem. Phys. 2010, 12, 2751-2757.

(18) Wong, B. M. J. Phys. Chem. C 2009, 113, 21921-21927.

(19) Camacho, C.; Niehaus, T. A.; Itami, K.; Irle, S. Chem. Sci. 2013, 4, 187-195. 
(20) Yagi, A.; Segawa, Y.; Itami, K. J. Am. Chem. Soc. 2012, 134, $2962-2965$.

(21) Omachi, H.; Nakayama, T.; Takahashi, E.; Segawa, Y.; Itami, K. Nat. Chem. 2013, 5, 572-576.

(22) Hirst, E. S.; Wang, F.; Jasti, J. Org. Lett. 2011, 13, 6220-6223.

(23) Golder, M. R.; Wong, B. M.; Jasti, R. Chem. Sci. 2013, 4, 42854291.

(24) Xia, J.; Golder, M. R.; Foster, M. E.; Wong, B. M.; Jasti, R. J. Am. Chem. Soc. 2012, 134, 19709-19715.

(25) Jasti, R.; Bertozzi, C. R. Chem. Phys. Lett. 2010, 494, 1-7.

(26) Wong, B. M. J. Phys. Chem. Lett. 2011, 2, 2702-2106.

(27) Nayyar, I. H.; Batista, E. R.; Tretiak, S.; Saxena, A.; Smith, D. L.; Martin, R. L. J. Chem. Theory Comput. 2013, 9, 1144-1154.

(28) Nayyar, I. H.; Batista, E. R.; Tretiak, S.; Saxena, A.; Smith, D. L.; Martin, R. L. J. Phys. Chem. Lett. 2011, 2, 566-571.

(29) Wu, C.; Malinin, S. V.; Tretiak, S.; Chernyak, V. Y. Nat. Phys. 2006, 2, 631-635.

(30) Li, H.; Chernyak, V. Y.; Tretiak, S. J. Phys. Chem. Lett. 2012, 3, 3734-3739.

(31) Bednarz, M.; Malyshev, V. A.; Knoester, J. J. Chem. Phys. 2004, $120,3827-3840$.

(32) Bednarz, M.; Malyshev, V. A.; Knoester, J. Phys. Rev. Lett. 2003, 91, 217401.

(33) Tretiak, S.; Saxena, A.; Martin, R. L.; Bishop, A. R. Phys. Rev. Lett. 2002, 89, 097402.

(34) Karabunarliev, S.; Baumgarten, M.; Bittner, E.; Mullen, K. J. Chem. Phys. 2000, 113, 11372-11381.

(35) Franco, I.; Tretiak, S. J. Am. Chem. Soc. 2004, 126, 1213012140.

(36) Clark, J.; Nelson, T.; Tretiak, S.; Cirmi, G.; Lanzani, G. Nat. Phys. 2012, 8, 225-231.

(37) Kasha, M. Discuss. Faraday Soc. 1950, 9, 14-19.

(38) Condon, E. U. Phys. Rev. 1928, 32, 858-872.

(39) Park, Y.; Kuo, C.-Y.; Martinez, J. S.; Park, Y.-S.; Postupna, O.; Zhugayevych, A.; Kim, S.; Park, J.; Tretiak, S.; Wang, H.-L. ACS Appl. Mater. Int. 2013, 5, 4685-4695.

(40) Frisch, M. J. et al. Gaussian 09, Revision A.01; Gaussian Inc.: Wallingford CT, 2009.

(41) Yanai, T.; Tew, D. P.; Handy, N. C. Chem. Phys. Lett. 2004, 393, 51-7.

(42) Korzdorfer, T.; Sears, J. S.; Sutton, C.; Bredas, J.-L. J. Chem. Phys. 2011, 135, 204107.

(43) Igumenshchev, K. I.; Tretiak, S.; Chernyak, V. Y. J. Chem. Phys. 2007, 127, 1-10.

(44) Wong, B. M.; Hsieh, T. H. J. Chem. Theory Comput. 2010, 6, 3704-3712.

(45) Hanwell, M. D.; Curtis, D. E.; Lonie, D. C.; Vandermeersch, T.; Zurek, E.; Hutchinson, G. R. J. Cheminf. 2012, 4, 17 http://avogadro. openmolecules.net.

(46) Nelson, T.; Fernandez-Aberti, S.; Chernyak, V.; Roitberg, A. E.; Tretiak, S. J. Phys. Chem. B 2011, 115, 5402-5414.

(47) Nelson, T.; Fernandez-Aberti, S.; Roitberg, A. E.; Tretiak, S. Acc. Chem. Res. 2014, 47, 1155-1164. 